\title{
A New-Type Swing Chamber Artillery Sealing Structure Optimization
}

\author{
Chen Longmiao* and Fu Qiang
}

School of Mechanical Engineering, Nanjing University of Science and Technology, Nanjing, Jiangsu, 210094, P.R. China

\begin{abstract}
To solve the extracting problem caused by the sealing structure design of a new-type swing chamber artillery, the sealing system was analyzed and the sealing reliability standard was established. Based on the multi-disciplinary design optimization platform and the large-scale nonlinear FEA software ABAQUS, with the residual contact force as optimized objective and the standard as constraint, the optimized simulation has been conducted to the sealing structure. The optimization results show that in case the sealing time changed not so much, $25.76 \%$ of the extracting force would be reduced. Experiments show that the success of the sealing systems provides a way for solving engineering-related problems.
\end{abstract}

Keywords: FEA, optimal design, seal, swing chamber.

\section{INTRODUCTION}

Compared with the traditional artillery, the new artillery with the sealing structure makes a prominent change, in which the swing chamber and the body of artillery are designed separately. The design brings a lot of benefits, such as the firing rate of artillery is soared, and the design of feeding mechanism is simplified. But at the same time, the design also caused the gas leak at the contact surface of swing chamber and the end body of artillery. The swing chamber artillery fired sunk ammunition which is in semicombustible cartridge case, and the structure of cartridge is between the traditional cartridge case and the combustible cartridge case [1]. Through the optimal design of semicombustible cartridge case, the problem for seal has been well solved, however, it results in the relationship between seal and extracting problems: On the one hand, the measures for increasing the length, thickness, reducing initial seal gaps of different sealing section can solve sealing problem. However it may be followed by the increasing of residual contact force between cartridge and swing chamber which leads to the difficulty of extracting for artillery. Therefore, how to design the sealing structure reasonably has become a key technology for the development of the new artillery.

Through the analysis of the sealing system, the key sealing area of the new-type artillery was found, and then the sealing reliable standard was established. By using Python language for Secondary Development of the large-scale nonlinear FEA software ABAQUS, the parametric model of sealing structure has been built, and the structure has been optimized. By comparing the situations before and after optimization, it can be seen that the residual contact force and the reliable sealing time are all coming with satisfactory results whether in simulation or through emission test.

\section{ANALYSIS OF THE SEALING STRUCTURE}

Sealing system structure of the new artillery swing chamber is shown in Fig. (1). The system mainly has three possible leakage channels, channel A, B and C, respectively.

Through the analysis of the sealing mechanism in the system, it is figured out that channel $\mathrm{A}$ and $\mathrm{C}$ both can realize the rapid sealing at a low pressure and the reliable sealing at a high pressure, but as for channel $\mathrm{B}$, the time of sealing or the reliability of the sealing depends on the structure design of the tail thin-walled segment of the semicombustible cartridge case. Through theoretical analysis and engineering experience, it is ensured that length, thickness of thin-walled segment and the initial gap between swing chamber and cartridge case are the important factors which affect the sealing conditions. It implies that the sealing of channel B is the key to the sealing system. So in this paper the moment that swing chamber contact with cartridge case is the judgment criteria which determines whether the sealing system is reliable or not [2-4].

\section{OPTIMIZATION ANALYSIS}

\subsection{Establishment of the Parametrical Finite Element Model}

\subsubsection{Model Simplification [5]}

In order to facilitate calculation, some necessary simplifications need to be done before the finite element parametric modeling.

(1) For the semi-combustible cartridge case, the geometric shape and boundary condition are symmetrical to the respective rotary axis, so is the 
action of displacement, stress and strain under loading. Therefore, the finite element model can be established according to the axisymmetric problems.
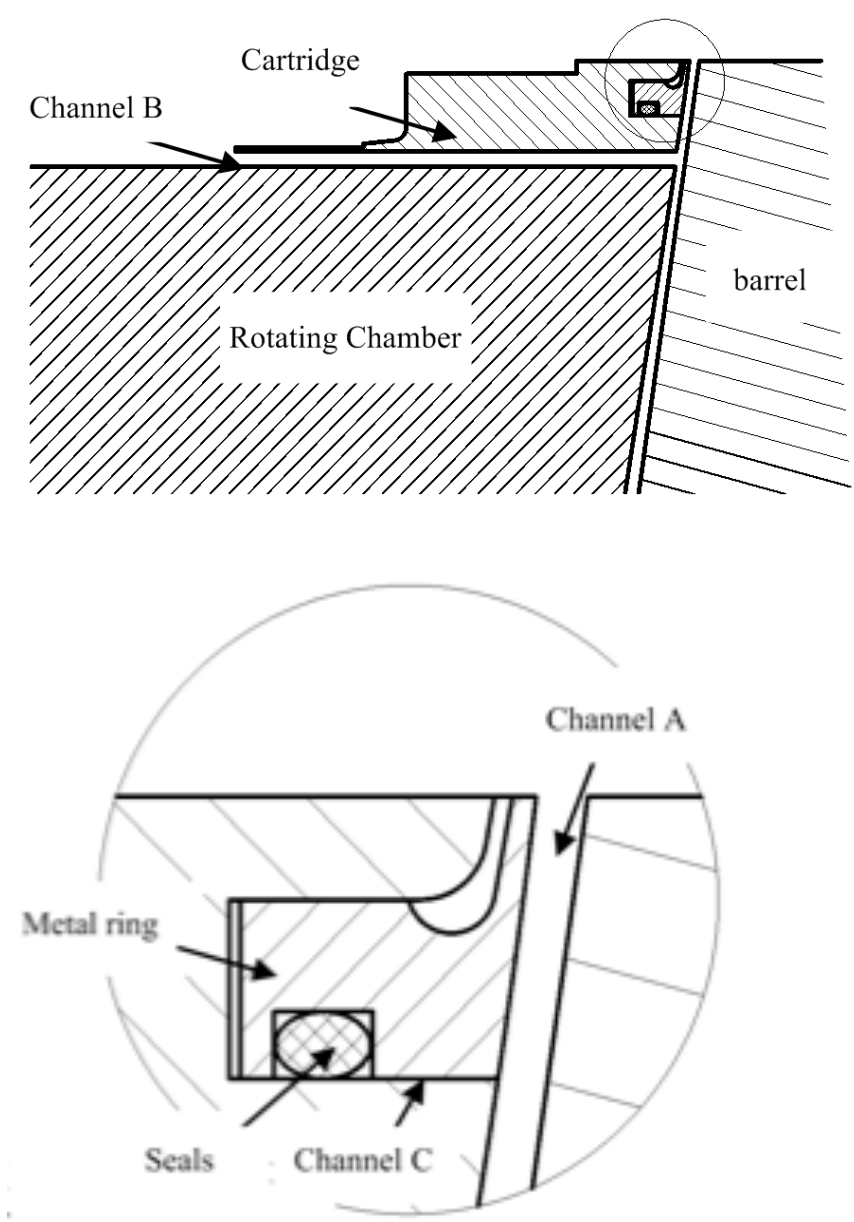

Fig. (1). Schematic diagram of seal structure.

(2) In order to analyze the effects that metal ring and rubber seal ring have on the contact condition between cartridge case and swing chamber, two kinds of finite element models are established (cartridge case with metal ring and rubber seal ring and cartridge case without metal ring and rubber seal ring). The calculation of the two kinds of models indicates that the error is only $1.25 \%$. Thus, for the convenience of calculation the metal ring and rubber sealing ring are omitted.

(3) The seal mechanisms of the semi-combustible cartridge case in the front and on the back are almost like each other. The sealed environment is harsher in the front due to the absence of function on the bottom. So, in this paper, the sealing problem will revolve around the front sealing conditions.

\subsubsection{Establishment of the Finite Element Model}

Taking the calculation accuracy and computational time into account, parts at the appropriate regions are split. On one hand, we refine grids in the contact and stress concentration areas. On the other hand, we will increase grid density in some non-contact area such as the chamber to lower the grids and the number of elements in non-key parts, which reduces the calculation time and improve the efficiency of the finite element calculation. Fig. (2). is for the computing grid model of sealing system.

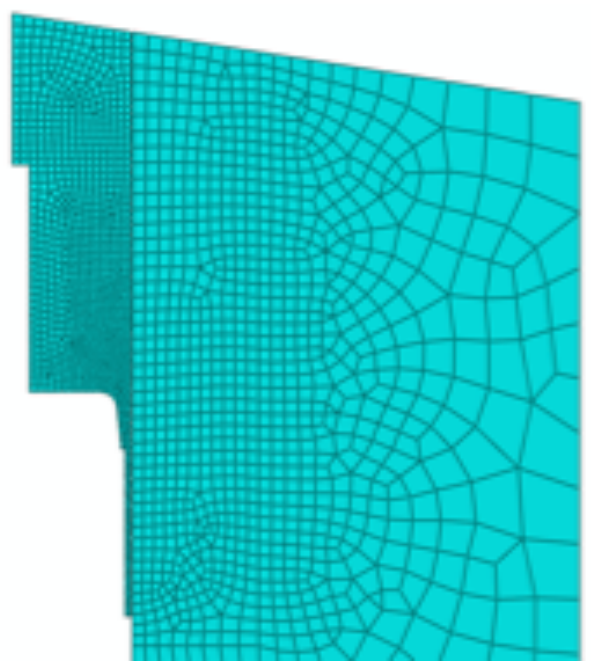

Fig. (2). Computation grid model of seal structure.

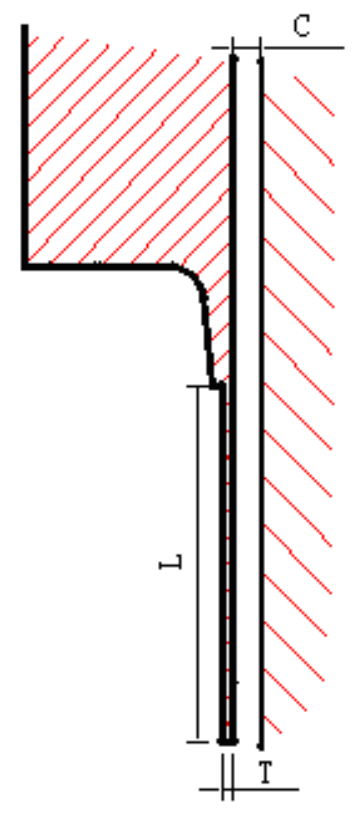

Fig. (3). Schematic diagram of the physical variables to optimize.

\subsubsection{Model Parameter Setting}

Parameterizing the model is the premise of realizing the automatic optimization process, and the essence of parameterizing the model can be regarded as the replacement of the fixed constants with the naming variables.

ABAQUS software is widely used to store and restore data, and abaqus.rpy belongs to the temporary file recording almost all the ABAQUS/CAE commands in an operation.

This paper adopts the abaqus.rpy file and edited in Python language, creating files available for debugging by ABAQUS/PDE [6]. This file which is the foundation of further optimization contains commands like modeling, 
parameter setting, meshing, boundary conditions setting and calculation results reading, etc.

\subsection{The Establishment of the Optimization Mathematical Model}

According to the theoretical calculation formula of extracting force model, extracting force is closely related to some parameters of cartridge and barrel chamber, such as material parameters, geometric structure size, clearance, etc. For semi-combustible cartridge case extracting force model, the elastic modulus of barrel chamber and semi-combustible, the coefficient of friction between the two kinds of materials, and the enhanced characteristics of Semi-combustible cartridge, these unknown variables are decided by determining the type of material, while the absolute value of Poisson's ratio is a constant under the condition of that the stress does not exceed proportional limit, and the maximum chamber pressure can be also regarded as constant under the premise of established interior ballistic indicators [7].

The size of barrel chamber are constrained by the general performance indices of artillery, and it can be regarded as constant. As is shown in Fig. (3), three design variables which have the greatest impact on the residual contact force can be determined: the length of the sealing section (L), the initial clearance $(\mathrm{C})$ and the thickness of the sealing section (T). At the same time, the earlier the cartridge and the sealing chamber begin to contact, the better sealing effect is. As a result, not only the reliable sealing standards in section 2 is regarded as constraints, but also the starting time of contact is treated as the secondary optimization goals. So, the optimization objective function in this paper can be expressed as

$$
\min F(X)=(f(t) \downarrow, S \downarrow)
$$

Among them: $S$-sealing time. Just at the moment, the cartridge and the sealing chamber begin to contact; $f(\mathrm{t})$ - the residual contact force between cartridge and swing chamber.

In order to give consideration to both calculation accuracy and computational efficiency, response surface method is employed in this paper. It means that building the agent model between the design variables and objective function through experimental design, then read data directly from the agent model just to replace real simulation. The ratio of finite element method and response surface calculation is 1:4. This paper used MOGAII, the initial population is 20 , which can enhance both the stability of the algorithm and maintain the population diversity, which provides an easy access to the global optimal solution. Maximum evolution generation is 100 , the crossover probability of the direction is 0.5 , the choosing probability is 0.05 , mutation probability is 0.1 , and mutation rate of DNA is 0.05 . The multidisciplinary optimization software has calculated for 2000 times. The whole time of optimization calculation is 17 hours and 5 minutes.

\subsection{Optimization Results and Analysis}

Due to the fact that there are three objective functions, the three-dimension bubble chart will be used to display them. As is shown in Fig. (4), horizontal axis, vertical axis and the bubble size respectively represent the size of the residual contact force, the time for establishing a sealing and the quality.

All design points in Fig. (4) are all feasible design points, and the pareto frontier points are those bubbles marked by circles outside.

The figure shows that it's impossible to make the size of the residual contact force, the time for establishing a sealing and the quality minimum at the same time.

Combined with the optimization target, the principle to select the final design point from the pareto frontier design points is determined: taking the reliable sealing as the premise, that is, the sealing time is close to or equal to the time before optimization, we will try to select the design point of which the residual contact force is the minimum, moreover, the quality of the seal section is acted as the subordinate reference index.

Based on this principle of selecting, the ID442 number design point is ultimately determined as the final design point.

As is shown in Table 1, the time of establishing a reliable sealing (the time of sealing) only changes by $0.31 \%$ through optimization, while the residual contact force is significantly reduced by $25.76 \%$, achieving the expected optimization goal.

The optimized structure analyzed by simulation shows that sealing condition changes a little in the entire shooting time domain and the contact stress between cartridge case and swing chamber decreases only in the last stage of sealing, which states the optimization process doesn't have much effect on the overall sealing performance. At the same time, residual contact force decreases a lot on the premise of reliable sealing in the process of live firing, which makes a satisfactory result.

The artillery firing test before sealing structure optimization show us some phenomena: plastic deformation occurs in the sealing section which can effectively seal gas, however, the residual contact stress is too large for the motor to drive the next projectile to push the residual parts of the semi-combustible cartridge case out, so it don't have the running fire condition (Fig. 5).

Using the results of this study to make some minor changes on the size of the seal segments, we do some single and running fire tests of which the chamber pressure is from $170 \mathrm{MPa}$ to $200 \mathrm{MPa}$. The tests show that all parts of the artillery are normal and no gas leak can be found on junction surfaces among swing chamber, barrel and semi-combustible cartridge case, which prove that sealing system is not only reliable but also can reduce residual contact force effectively. So the results from this paper successfully solve a key technical problem of the artillery systems.

\section{CONCLUSION}

The application of the finite element analysis and optimization design ideas into the design of new artillery sealing structure not only achieves the goals as a reliable sealing and reduced residual contact force, but also provides a good reference for the related problems in this field. 


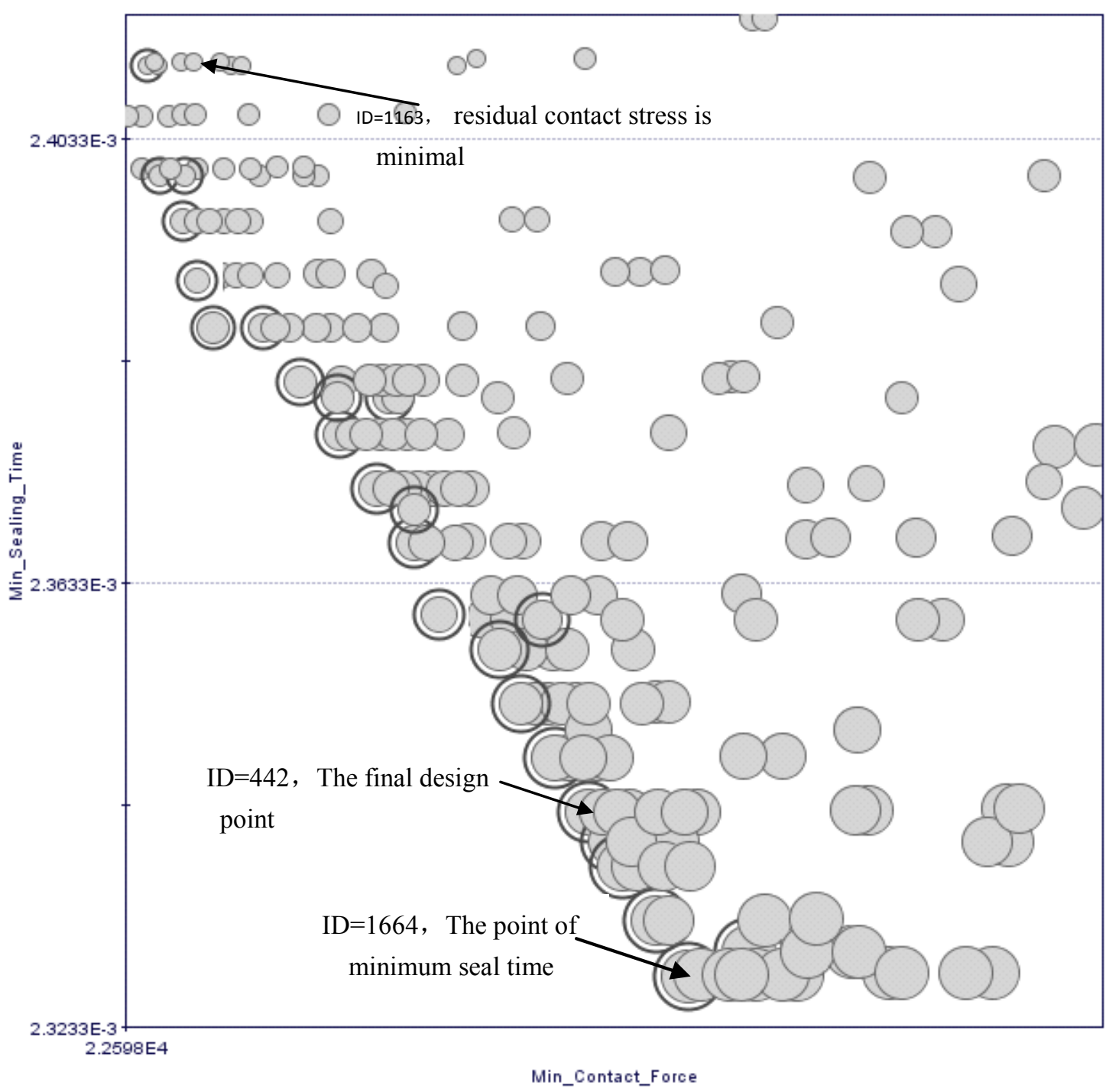

Fig. (4). The three-dimension bubble chart of objective function for seal structure.

Table 1. Optimization index's comparison chart before and after optimization.

\begin{tabular}{|c|c|c|c|c|c|}
\hline & Thickness (mm) & Length (mm) & Gap (mm) & Residual Contact Stress (KN) & The Time for Starting Seal (ms) \\
\hline \hline Upper limit & 1.20 & 30.50 & 0.30 & $/$ & $/$ \\
\hline Lower limit & 0.96 & 29.50 & 0.15 & $/$ & 33.050 \\
\hline Before optimization & 1.00 & 30.00 & 0.28 & 24.536 & 2.3500 \\
\hline After optimization & 1.05 & 29.51 & 0.30 & 2.3427 \\
\hline
\end{tabular}
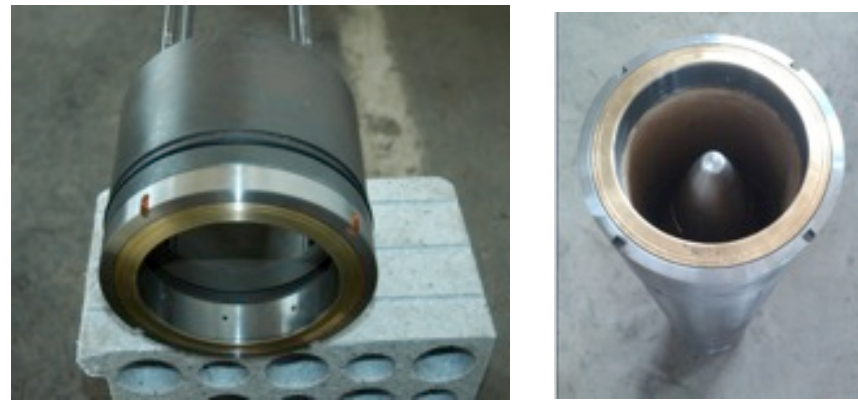

\section{CONFLICT OF INTEREST}

The authors confirm that this article content has no conflict of interest.

\section{ACKNOWLEDGEMENTS}

The authors would like to thank for financial support the National Natural Science Foundation of China (51205207).

Fig. (5). Optimized ammunition system of sealing structure. 


\section{REFERENCES}

[1] J.B. Wang, L.S. Fan, J. Wen, and L.T. Xu, "Evaluation of storage reliability for semi-combustible cartridge," Initiators \& Pyrotechics, vol. 3, pp. 5-8, 1999

[2] H. Xu, Sealing, Beijing: Metallurgical Industry Press, pp. 20-30, 1999.

[3] Q. Fu, "Optimization Design of a Certain Type Semi-Combustible Cartridge Case Sealing Structure", Ms thesis, Nan Jing Uni-versity of Science and Technology, 2012.

[4] H. Zhang, and Y.H. Zhou, "A seal design under transient high pressure condition", Lubrication Engineering, vol. 5, pp. 132-134, 2006.
[5] X. Zhang, and X.Y. Zhang, "Finite element analysis of contact problem on the new combined barrel seal structure", Lubrication Engineering, vol. 11, pp. 69-72, 2008.

[6] T.S. Zhong, F. Wei, Z. Wang, and Y.H. Zhi, "Second development for fore treatment of ABAQUS using python language", Journal of Zhengzhou University, vol. 1, pp. 60-64, 2006.

[7] H. Wang Q. Han, and M.M. Tao, "Strength analysis of welded cartridges and shells force calculation", Technology Foundation of National Defence, vol. 7, pp. 54-58, 2009.

(C) Longmiao and Qiang; Licensee Bentham Open.

This is an open access article licensed under the terms of the Creative Commons Attribution Non-Commercial License (http://creativecommons.org/licenses/by-nc/3.0/) which permits unrestricted, non-commercial use, distribution and reproduction in any medium, provided the work is properly cited. 